\title{
HEAT RELEASE DYNAMICS MODELING FOR COMBUSTION INSTABILITY ANALYSIS OF KINETICALLY CONTROLLED BURNING
}

\author{
Sungbae Park, Anuradha Annaswamy and Ahmed Ghoniem \\ Department of Mechanical Engineering \\ Massachusetts Institute of Technology
}

\begin{abstract}
$\underline{\text { Abstract }}$
We present a heat release dynamics model which utilizes a well-stirred reactor (WSR) model and one-step kinetics to describe the unsteady combustion process. The model incorporates the linearized mass and energy equations to describe the response of the reactor to external perturbations, and is cast in the form of a first order filter. The model is able to predict the phase between the mass flow rate oscillations and the resulting heat release fluctuations, as function of the operating conditions, e.g., the mean equivalence ratio and mean mass flow rate. The model predicts a sudden shift in phase in the region between the maximum reaction rate and the blow-out limit. We show that this phase change may trigger combustion instability. We use this novel model to predict combustion instability conditions in high swirl combustion, and demonstrate that these predictions agree qualitatively with experimental studies.
\end{abstract}




\section{Introduction}

Combustion in high performance engines utilizes strong swirl, recirculation and interacting jets to enhance the mixing rate of the fuel, air and products, and hence maximize the burning rate. The ideal limit for these systems is often modeled as a wellstirred reactor [1], in which the mixing rate is faster than the fuel conversion rate, and products exit the reactor at their interior uniform state. The operation of a well-stirred reactor is governed by a characteristic residence time, $\tau_{r e s}$, which is the nominal time the reactants spend inside the reactor;

$$
\tau_{\text {res }}=\frac{\rho_{i} V}{\dot{m}_{i}}
$$

where $\rho_{i}$ is the density of the reactant, $V$ is the reactor volume, and $\dot{m}_{i}$ is the mass flow rate at the inlet. Stable operation is achieved when the residence time is larger than the characteristic chemical time; otherwise blow-out should be expected.

Combustion instability, resulting from coupled heat release-pressure oscillations, has been suspected to occur when oscillations in the mass-flow rate, equivalence ratio, inlet temperature and pressure, etc., occur at the same time-scale. However, the mechanisms that support the positive coupling between the heat release dynamics and acoustic perturbations have not yet been investigated or modeled thoroughly. The condition under which a combustion system becomes unstable has been expressed in terms of the Rayleigh criterion [2]:

$$
\frac{\partial}{\partial t} \int_{0}^{L} e^{\prime} A d x=\frac{\gamma-1}{\overline{\rho \bar{c}^{2}}} \int_{0}^{L} p^{\prime} q^{\prime} A d x-\Delta_{L}\left(E^{\prime} A\right)-\dot{\Phi}>0
$$


where $e^{\prime}=\frac{p^{\prime 2}}{2 \overline{\rho c}^{2}}+\frac{\bar{\rho}{ }^{\prime 2}}{2}$ and $E^{\prime}=P^{\prime} \vec{v}^{\prime}$ are the acoustic energy density and acoustic energy flux, respectively, $\rho^{\prime}$ is the perturbation in the density of the unburned mixture, $A$ is the cross-sectional area of the combustor, $\dot{\Phi}$ is the perturbation in the rate of energy dissipation, $x$ and $t$ are the distance and time, respectively, and $\Delta_{L}$ signifies the difference over the combustor length $L$. The conclusion drawn from this mathematical condition is that a combustion system becomes unstable when the heat release increases at a moment of a rise in pressure, i.e., $\angle\left(q^{\prime}-p^{\prime}\right) \leq 90^{\circ}$. The Rayleigh criterion also shows that acoustic energy depends on the dissipation in the system, and hence the gain in the $\left(p^{\prime}-q^{\prime}\right)$ relationship also plays an important role in determining the characteristics of instability.

Combustion instability has been modeled using a well-stirred reactor and one-step kinetics by Richards et al. [3], Janus and Richards [4], Lieuwen et al. [5], and Lieuwen and Zinn [6]. Richards et al. [3] investigated the effect of heat loss, flow rate and friction in a tailpipe of a pulse combustor. The governing flow equations were reduced to a set of ODEs assuming a well-mixed combustion zone and choked inlet flow. The authors showed that the simulation results of the ODEs agree qualitatively with the experimental data. A similar approach was used by Janus and Richards [4] for a premixed combustor. In that study, the authors showed that the model could predict the effect of the inlet temperature and open loop control by comparing the simulations with experimental results. Lieuwen et al. [5] investigated the impact of the equivalence ratio oscillation on the heat release. Given a perturbation in the equivalence ratio, as the mean equivalence ratio is decreased, they show that a well-stirred reactor model yields an increase in the magnitude of the corresponding heat release perturbations. In [6], the same model was 
coupled with acoustics and a convective time delay for the equivalence ratio perturbation, and instability was predicted over a range equivalence ratio of 0.6-1.

In this work, we investigate the linear response of a WSR model to the mass flow rate, or residence time oscillations, using one-step kinetics. We show that as the mean equivalence ratio or the mean residence time approach the blow-out limit, the operating point may transition from stability to instability due to a sudden phase change between pressure and heat release oscillations.

In Section II, a linearized heat release dynamics model is developed and the resulting model is examined in light of the physics of a WSR. In Section III, we investigate the impact of the operating conditions, e.g., mean equivalence ratio and mean mass flow rate on the properties of the model. In section IV, coupling with acoustics is described and conditions of thermoacoustic instability are investigated. Predictions are then compared with experimental results and evidence supporting the WSR model results are summarized in Section V. In Section VI, we assemble a model for the LSU experiment and compare our prediction regarding impact of operating conditions with the results of that experiment. Conclusions are summarized in Section VII. 


\section{Analytical Modeling of the WSR}

\section{II.1 Governing Equations}

The governing equations of a well-stirred reactor are obtained using the conservation laws and a set of reaction-rate equations. The conservation equations of the mass, energy and species in the WSR are given by:

Mass Conservation: $\frac{d M}{d t}=\dot{m}_{i}-\dot{m}$,

Energy Conservation: $\frac{d E}{d t}=\dot{m}_{i} h_{i}-\dot{m} h+\dot{Q}_{r}$,

Species Conservation: $\frac{d M_{k}}{d t}=\dot{m}_{i} Y_{k, i}-\dot{m} Y_{k}-\dot{W}_{k}$,

where $M, E$, and $M_{k}$ are a total mass, energy and mass of species $k$ inside the combustor, respectively, $\dot{Q}_{r}$ is the heat release rate due to the chemical reaction, $\dot{W}_{k}$ is a consumption rate of species $k, \dot{m}$ is the mass flow rate, $h$ is the enthalpy, $Y$ is the mass fraction, and subscript $i$ refer to the inlet condition. We assume that the condition at the exit are the same as inside, consistent with the assumption that mixing is much faster than the chemical reaction. Equation (4) can be written for all species; e.g., $\mathrm{C}_{n} \mathrm{H}_{m}, \mathrm{O}_{2}, \mathrm{CO}_{2}$, $\mathrm{H}_{2} \mathrm{O}$, etc. In case of one-step kinetics, one differential equation is sufficient and the mass fractions of other species are related by stoichiometry. Equation (2) and (3) can be simplified as follow:

$$
\rho V c_{p} \frac{d T}{d t}-V \frac{d p}{d t}=\dot{m}_{i} c_{p}\left(T_{i}-T\right)+\dot{Q}_{r}
$$


where $\rho$ is the density of the mixture, $V$ is the volume, $c_{p}$ is the specific heat, $T$ is temperature, and $p$ is the pressure. In deriving equation (5), we assume that the $c_{v}$, $c_{p}$ and $V$ are constants. The $V \frac{d p}{d t}$ term can be expressed as a function of $T$ using the inlet and exit conditions, and the ideal gas law. Assuming that the pressure oscillations are weak, the pressure energy term is negligible, and equation (5) reduces to

$$
\rho V c_{p} \frac{d T}{d t}=\dot{m}_{i} c_{p}\left(T_{i}-T\right)+\dot{Q}_{r}
$$

Using equation (2), equation (4) reduces to

$$
\rho V \frac{d Y_{k}}{d t}=\dot{m}_{i}\left(Y_{k, i}-Y_{k}\right)-\dot{W}_{k}
$$

The source terms, $\dot{Q}_{r}$, and $\dot{W}_{k}$ for the fuel, can be represented as function of $Y$ and $T$ using a one-step kinetics mechanism [7] as follows:

$\dot{W}_{f}=A_{f} V\left(\rho Y_{f}\right)^{n_{f}}\left(\rho Y_{o_{2}}\right)^{n_{o_{2}}} \exp \left(\frac{-T_{a}}{T_{o}}\right)$ and $\dot{Q}_{r}=\Delta h_{r} \dot{W}_{f}$

where $A_{f}$ is the frequency factor, $\Delta h_{r}$ is the enthalpy of reaction (measured per unit mass of fuel), and $T_{a}=E_{a} / R$ where $E_{a}$ is activation energy and $R$ is the gas constant.

At a fixed $\phi, Y_{O_{2}}$ and $Y_{f}$ are related by the stoichiometric mass ratio $\psi_{s}$ as follows:

$$
Y_{O_{2}}=\frac{1}{\psi_{s}} Y_{f}+\left(Y_{O_{2}}-\frac{1}{\psi_{s}} Y_{f}\right)
$$

Near stoichiometric conditions, $Y_{O_{2}} \approx \frac{1}{\psi_{s}} Y_{f}$, and far from the stoichiometry, i.e., in a fuel lean mixture, $Y_{\mathrm{O}_{2}} \approx$ const. In a fuel lean mixture, $\rho^{n_{f}}$ can taken as a constant around the equilibrium point because the strongest dependence of the reaction rate on temperature comes from exponential term. Therefore, equation (8) can be simplified to 


$$
\dot{Q}_{r}=A_{f}^{\prime} \Delta h_{r} V \rho^{n} Y^{n} \exp \left(\frac{-T_{a}}{T_{o}}\right)
$$

where $Y=Y_{f}$ and $n=n_{f}+n_{o_{2}}$ at near stoichiometric condition, and $n=n_{f}$ under fuel-lean condition.

\section{II.2 Linearized Heat Release Model}

While the dynamics of a well-stirred reactor can be investigated by integrating these nonlinear ODEs directly, a linearized model makes it possible to examine its properties, such as the blow-out limit, and the gain and phase relations between the heat release rate and mass flow perturbations. A linear heat release model can be obtained from equations (6), (7) and (10) assuming small perturbations around a steady state. In deriving the linear heat release model, it is assumed that the air and the fuel lines are choked. Therefore, equivalence ratio oscillations are absent, while the mass flow rate and temperature oscillations in the combustion zone are the forcing terms of the heat release model. The dependent variables $T, Y, \rho$ and $\dot{m}_{i}$ are represented using steady-state and perturbation terms, e.g. $Y=\bar{Y}+Y^{\prime}$. The linearized reaction rate equation (10) is:

$$
\dot{Q}_{r}^{\prime}=A_{f}^{\prime} \Delta h_{r} V\left[n \bar{\rho}^{n-1} \rho^{\prime} \bar{Y}^{n} \exp \left(\frac{-T_{a}}{\bar{T}}\right)+n \bar{\rho}^{n} \bar{Y}^{n-1} Y^{\prime} \exp \left(\frac{-T_{a}}{\bar{T}}\right)+\bar{\rho}^{n} \bar{Y}^{n} \frac{T_{a}}{\bar{T}^{2}} \exp \left(\frac{-T_{a}}{\bar{T}}\right) T^{\prime}\right] .
$$

Moreover, $\rho^{\prime}$ is expressed in terms of $T^{\prime}$ (assuming constant pressure and molecular weight),

$$
\rho^{\prime}=-\bar{\rho} \frac{T_{o}^{\prime}}{\bar{T}_{o}}
$$

Using equation (11) and (12), one can linearize equations (6) and (7) as 


$$
\begin{aligned}
\bar{\rho} V c_{p} \frac{d T^{\prime}}{d t}= & \dot{\bar{m}} c_{p}\left(-T^{\prime}\right)+\dot{m}^{\prime} c_{p}\left(T_{i}-\bar{T}\right)+A_{f}^{\prime} \Delta h_{r} V \times \\
& {\left[-n \bar{\rho}^{n} \bar{Y}^{n} \exp \left(\frac{-T_{a}}{\bar{T}}\right) \frac{T^{\prime}}{\bar{T}}+n \bar{\rho}^{n} \bar{Y}^{n-1} Y^{\prime} \exp \left(\frac{-T_{a}}{\bar{T}}\right)+\bar{\rho}^{n} \bar{Y}^{n} \frac{T_{a}}{\bar{T}^{2}} \exp \left(\frac{-T_{a}}{\bar{T}}\right) T^{\prime}\right], }
\end{aligned}
$$

$$
\bar{\rho} V \frac{d Y^{\prime}}{d t}=\dot{\bar{m}}\left(-Y^{\prime}\right)+\dot{m}^{\prime}\left(Y_{i}-\bar{Y}\right)-A_{f}^{\prime} V \times
$$

$$
\left[-n \bar{\rho}^{n} \bar{Y}^{n} \exp \left(\frac{-T_{a}}{\bar{T}}\right) \frac{T_{o}^{\prime}}{\bar{T}}+n \bar{\rho}^{n} \bar{Y}^{n-1} Y^{\prime} \exp \left(\frac{-T_{a}}{\bar{T}}\right)+\rho^{n} \bar{Y}^{n} \frac{T_{a}}{\bar{T}^{2}} \exp \left(\frac{-T_{a}}{\bar{T}}\right) T^{\prime}\right]
$$

Since the ratio $\left(\frac{T_{i}^{\prime}}{\bar{T}_{i}}\right) /\left(\frac{\dot{m}^{\prime}}{\dot{m}}\right)=M_{a}(\gamma-1)$, for low Mach number flows, we neglected $T_{i}^{\prime}$ in equation (13). Using Laplace transforms of equations (11), (13), and (14), we obtain the following linear heat release rate model:

$$
\dot{Q}_{r}^{\prime}=J(s) \dot{m}^{\prime}=\frac{\beta}{s+\alpha} \dot{m}^{\prime}
$$

where

$\alpha=\frac{\dot{\bar{m}}}{\bar{\rho} V}\left[1+n \frac{\left(\bar{T}-T_{i}\right)}{\bar{T}}-\frac{\left(\bar{T}-T_{i}\right)}{\bar{T}^{2}} T_{a}+n \frac{\left(Y_{i}-\bar{Y}\right)}{\bar{Y}}\right]$

and

$\beta=A_{f}^{\prime} \Delta h_{r} \bar{\rho}^{n-1} \bar{Y}^{n} \exp \left(\frac{-T_{a}}{\bar{T}}\right)\left[n \frac{\left(\bar{T}-T_{i}\right)}{\bar{T}}-\frac{\left(\bar{T}-T_{i}\right)}{\bar{T}^{2}} T_{a}+n \frac{\left(Y_{i}-\bar{Y}\right)}{\bar{Y}}\right]$

Note that $J(s)$ is a first order filter.

The cut-off frequency $\alpha$ and the static gain $\beta$ of the linear model are functions of the mean residence time, the equivalence ratio, and the inlet temperature. At a fixed equivalence ratio, if the residence time is much larger than the chemical reaction time, almost all the fuel is burnt, i.e., $\bar{Y} \approx 0$. In this case, $\alpha$ and $\beta$ are much larger than the 
acoustic frequency (due to the $\bar{Y}$ term in the denominator in equation (16) and (17)), and the heat release responses instantaneously to the acoustic perturbations. As the residence time decreases, the unburned fuel $\bar{Y}$ increases, so the values of $\alpha$ and $\beta$ decrease. Moreover, the change of the residence time affects the equilibrium temperature $\bar{T}$. As the residence time decreases, the equilibrium temperature $\bar{T}$ decreases, while $\alpha$ and $\beta$ change from positive to negative values because of the $-\frac{\left(\bar{T}_{o}-T_{i}\right)}{\bar{T}_{o}^{2}} T_{a}$ term. When $\alpha$ becomes negative, the heat release model itself becomes unstable since a perturbation grows exponentially as $e^{-\alpha t}$. The system is critically stable when $\alpha=0$. As we will see in the next section, this corresponds to blow-out. The value of $\bar{T}$ which leads to $\alpha=0$ in equation (16) is defined as $T^{*}$ which is the blow-out temperature; $T^{*}$ satisfies the following equation:

$1+n \frac{\left(T^{*}-T_{i}\right)}{T^{*}}-\frac{\left(T^{*}-T_{i}\right)}{\left(T^{*}\right)^{2}} T_{a}+n \frac{\left(Y_{i}-\bar{Y}\right)}{\bar{Y}}=0$

Equation (15) shows that when $\beta$ changes sign, it introduces a $180^{\circ}$ phase change between $\dot{m}^{\prime}$ and $\dot{Q}_{r}^{\prime}$. If the heat release dynamics is coupled with acoustics, this phase change may trigger a thermoacoustic instability as an out-of-phase relationship between $\left(p^{\prime}, q^{\prime}\right)$ becomes in-phase. That is, at $\beta=0$, the system can transition from stability to instability. This thermoacoustic driven instability is different from the instability of the flame dynamics itself, which is defined by the sign of $\alpha$ in the above paragraph. The critical value of $\bar{T}$ which corresponds to $\beta=0$ in equation (17) is denoted as $T^{* *}$, and is determined from 
$n \frac{\left(T^{* *}-T_{i}\right)}{T^{* *}}-\frac{\left(T^{* *}-T_{i}\right)}{\left(T^{* *}\right)^{2}} T_{a}+n \frac{\left(Y_{i}-\bar{Y}\right)}{\bar{Y}}=0$

As will be shown in the next section, $\beta=0$ corresponds to burning at the maximum heat release rate. Equations (18) and (19) are similar expect for the extra " 1 " in equation (18). Based on this, one expects $\beta$ to become negative before $\alpha$ as the residence time decreases. Therefore, just before blow out $(\alpha=0)$, the heat release experiences a phase change. That is, the onset of thermoacoustic instability may occur before blow-out.

The change of the equivalence ratio at a fixed residence time also changes the equilibrium temperature $\bar{T}$, thereby affecting $\alpha$ and $\beta$. One can expect that $\alpha$ and $\beta$ become negative as the equivalence ratio decreases due to the drop of the equilibrium temperature $\bar{T}$. Therefore, the linearized model shows that by decreasing the residence time or the equivalence ratio, one expects phase change or blow-out to occur.

\section{II.3 Physical Insight into the WSR Model}

The heat release dynamics model presented in Section II. 2 has two parameters $\alpha$ and $\beta$. To gain insight into the meaning of $\alpha$ and $\beta$, we examine the critical steady state response of the WSR. We define

$\dot{Q}_{f}=\dot{m}_{i} c_{p}\left(T-T_{i}\right)$ 
as the energy added to the flow across the reactor, and draw $\dot{Q}_{r}$ and $\dot{Q}_{f}$ as $\dot{m}$ changes, as shown in Figure $1^{1}$. The equilibrium or steady-state temperature is determined by the intersection of two curves. As known in the well-stirred reactor theory, three solutions exist; hot and cold stable solutions and an unstable hot solution. As the slope of the $\dot{Q}_{f}$ curve increases due to an increase in mass flux (or decrease of the residence time), the two hot solutions collapse onto one. There is no hot solution for higher values of mass flux. Therefore, the equilibrium point in Figure 1 where $\dot{Q}_{f}$-line becomes tangent to the $\dot{Q}_{r}$-curve is a critically stable point, and it can be calculated by solving the following equations:

$$
\dot{Q}_{r}=\dot{Q}_{f} \text { and } \frac{d \dot{Q}_{r}}{d T}=\frac{d \dot{Q}_{f}}{d T}
$$

The solution of these equations is given by equation (18), indicating that $\alpha=0$ captures the static blowout limit.

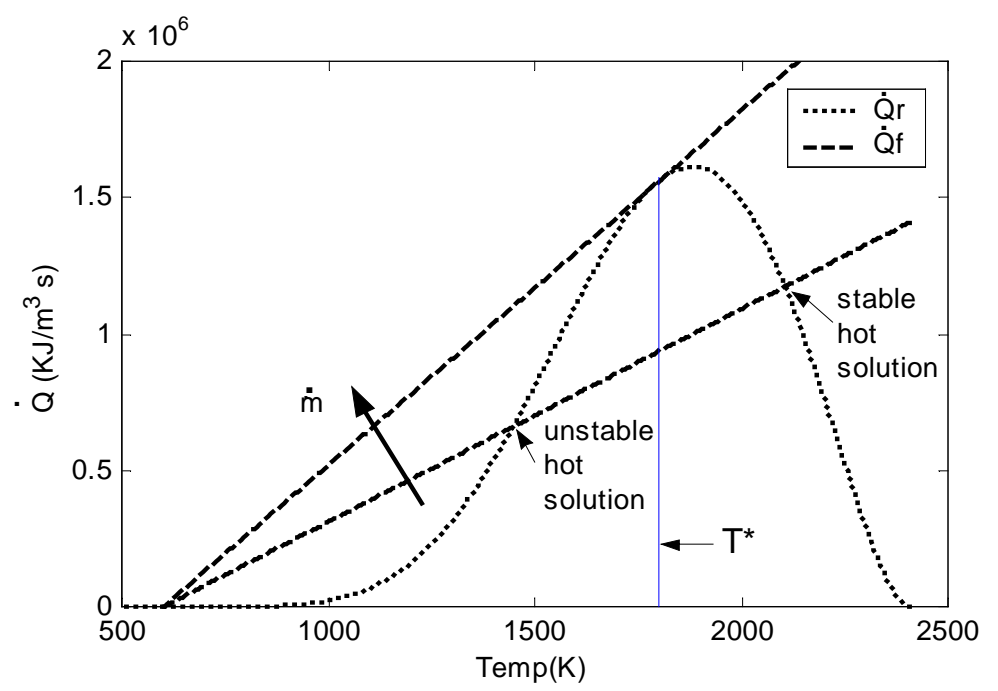

Figure 1 Definition of the blow-out Temperature $T^{*}$. Data are for $\phi=0.8$, $\dot{\bar{m}} / V=1040 \mathrm{~kg} / \mathrm{m}^{3} \mathrm{~s}$ and $T^{*}=1800 \mathrm{~K}$.

\footnotetext{
${ }^{1}$ All the Figures in this paper are calculated for $C_{2} H_{6}$, for which $A_{f}=4.24 \cdot 10^{8}, n_{f}=0.1, n_{O_{2}}=1.65$, $T_{a}=15098 \mathrm{~K}$ and $T_{i}=600 \mathrm{~K}$.
} 
Another critical point exists in $\dot{Q}_{r}$-curve. It occurs when $\dot{Q}_{r}$ reaches a maximum, as shown in Figure 2. The condition corresponding to maximum heat release rate,

$$
\frac{d \dot{Q}_{r}}{d T}=0
$$

is shown in Figure 2. The equation defining this temperature is exactly the same as $T^{* *}$ obtained from equation (19). The Figure shows that for $T>T^{* *}\left(\dot{m}_{i}^{\prime}, \dot{Q}_{r}^{\prime}\right)$ are in-phase; however, for $T^{*}<T<T^{* *}$, their phase changes by $180^{\circ}$. This is confirmed in Figure 3, where the equilibrium solution corresponds to $T^{*}<T<T^{* *}$. For $T>T^{* *}$, as the mass flow rate, $\dot{m}_{i}$ increases $\dot{Q}_{r}$ also increases, i.e., $\left(\dot{m}_{i}^{\prime}, \dot{Q}_{r}^{\prime}\right)$ are in-phase. On the other hand, for $T<T^{* *}$, as the mass flow rate, $\dot{m}_{i}$, increases, $\dot{Q}_{r}$ decreases, indicating an out-of-phase relation.

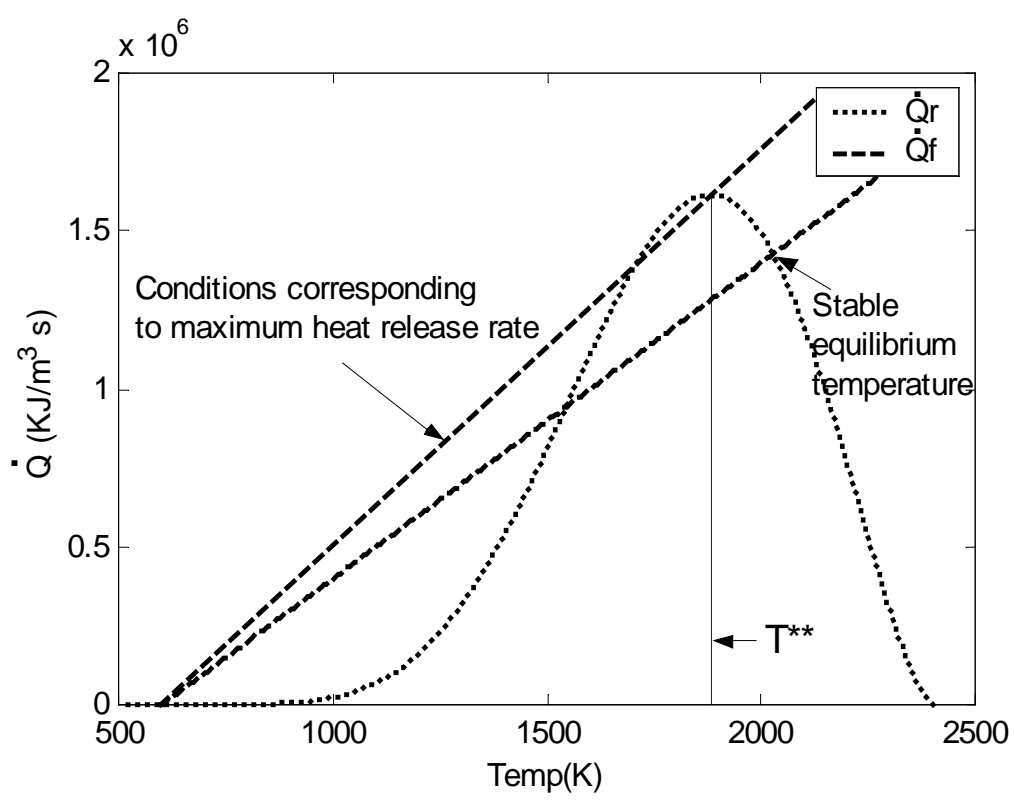

Figure 2 Plot showing conditions for in-phase relation between $\dot{Q}_{r}^{\prime}$ and $\dot{m}^{\prime}$, corresponding to $T>T^{* *}$. Data are for $\phi=0.8, \dot{\bar{m}} / V=800 \mathrm{~kg} / \mathrm{m}^{3} \mathrm{~s}$ and $T^{* *}=1882 \mathrm{~K}$. 


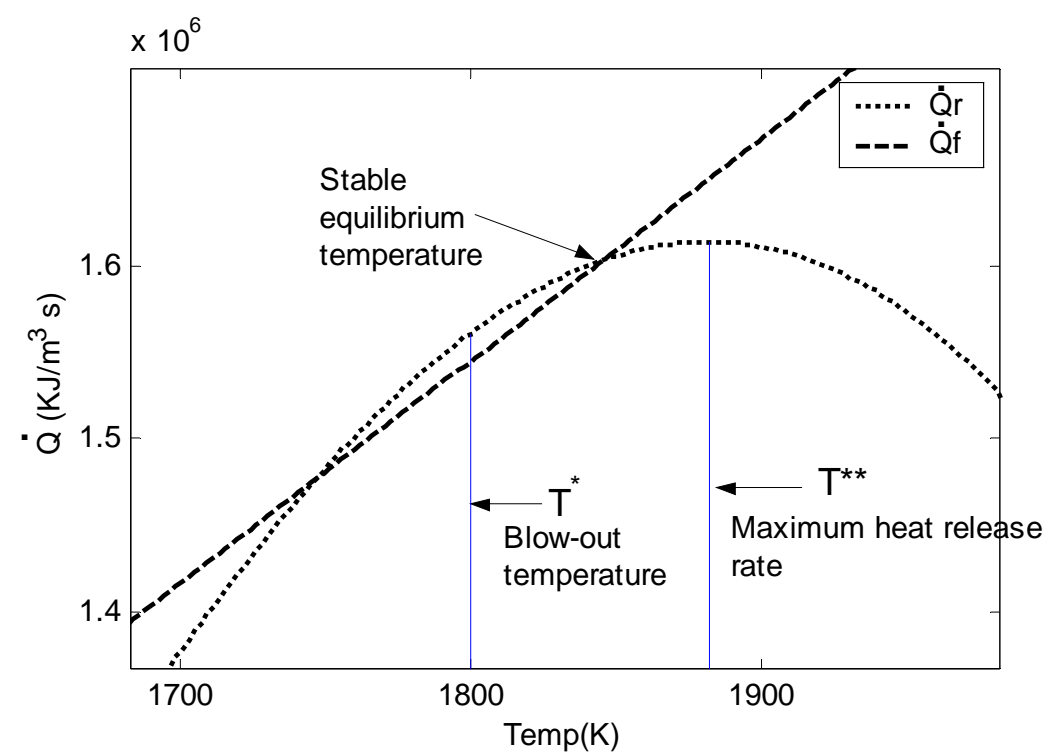

Figure 3 Plot showing conditions for out-of-phase relation between $\dot{Q}_{r}^{\prime}$ and $\dot{m}^{\prime}$, corresponding to $T^{*}<T<T^{* *}$. Data are for $\phi=0.8, \dot{\bar{m}} / V=1030 \mathrm{~kg} / \mathrm{m}^{3} \mathrm{~s}, T^{*}=1800 \mathrm{~K}$ and $T^{* *}=1882 K$.

Therefore, the phase between $\left(\dot{m}_{i}^{\prime}, \dot{Q}_{r}^{\prime}\right)$ changes by $180^{\circ}$ as the point of the maximum heat release rate is crossed. In Figure 2 and 3, the equilibrium condition shifts due to the change of the residence time (mass flow rate), which also leads to a phase change. Changing the equivalence ratio also can introduce phase change, as shown in Figure 4. As the equivalence ratio decreases, $\dot{Q}_{r}$ curve moves down causing the equilibrium point to cross the maximum heat release point. We conclude that a phase change of $180^{\circ}$ occurs either by decreasing the residence time, or equivalence ratio, in the regions between the maximum heat release point and the blow-out point.

In summary, the heat release dynamics is modeled as a first-order filter with a transfer function $J(s)$ given by equation (15). It is worth noting that even with such a simple form, the heat release model is capable of capturing blow-out, and the transition across 
the maximum heat release rate point. The first-order filter is able to characterize both of these characteristics through the two degrees of freedom $\alpha$ and $\beta$ which are parameters of the transfer function $J(s)$.

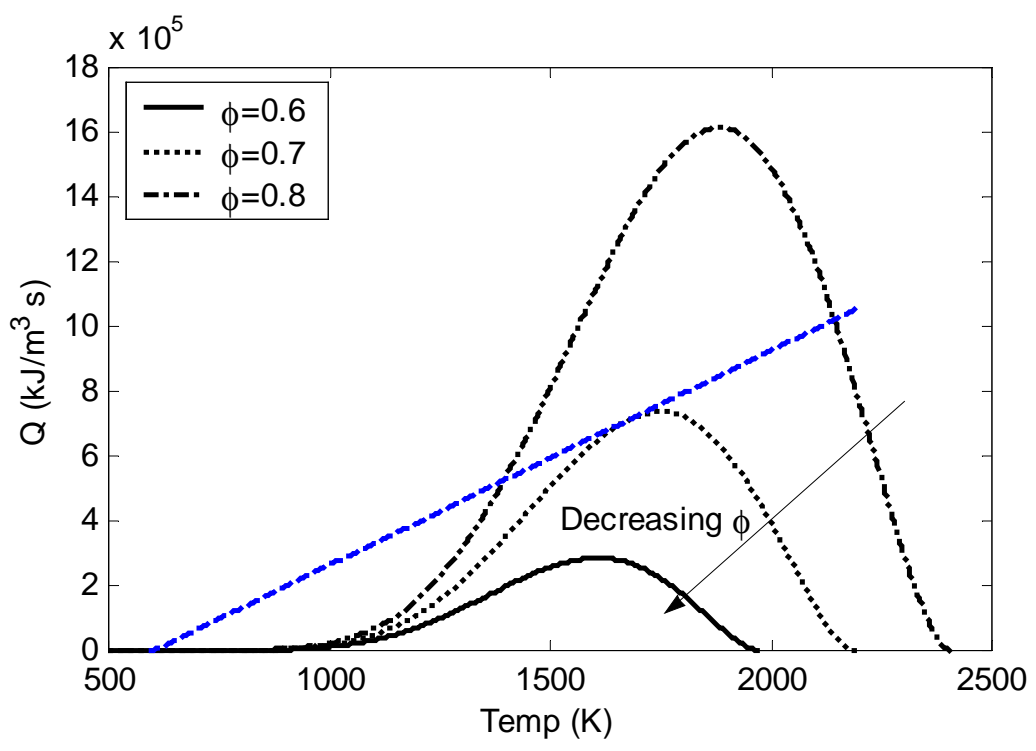

Figure 4 Plot showing the effect of $\phi$ on $\dot{Q}_{r}$. Data are for $\dot{\bar{m}} / V=530 \mathrm{~kg} / \mathrm{m}^{3} \mathrm{~s}$.

\section{Impact of Operating Conditions on the WSR Dynamics.}

The heat release model, $J(s)$, describes the linearized dynamics around a fixed operating condition. The operating condition is determined by $\phi, \dot{\bar{m}}$, and $T_{i}$. While the structure of the heat release model does not change as the operating condition changes, its parameters, the gain $\beta$ and the cut-off frequency $\alpha$, depend on the $\phi, \dot{\bar{m}}_{i}$ and $T_{i}$ through equations (16) and (17). We now show how these quantities change with $\phi$ and 
$\dot{\bar{m}}$ for $T_{i}=600 \mathrm{~K}$. Figure 5 depicts the impact of $\dot{\bar{m}}$ on $\alpha$ and $\beta$ at a fixed equivalence ratio. For values of $\dot{\bar{m}} / V$ is less than $700 \mathrm{~kg} / \mathrm{m}^{3} s$, the cutoff frequency $\alpha$ is about $3 \mathrm{khz}$. In this region, the heat release model $J(s)$ responds to the acoustic perturbation instantaneously when the frequency of the latter is of the order of a hundred Hz. As $\dot{\bar{m}} / V$ increases, $\beta$ becomes negative beyond the maximum heat release rate point. Around this area, $\alpha$ is close to the acoustic frequency. For a narrow range of $\dot{m} / V$, the phase between $\dot{Q}_{r}^{\prime}$ and $\dot{m}^{\prime}$ changes by $180^{\circ}$. Figure 6 shows the effect of the equivalence ratio on $\alpha$ and $\beta$ at a fixed mass flow rate. As the equivalence ratio decreases, $\alpha$ and $\beta$ decrease. In a narrow range of equivalence ratio from 0.7 to $0.705, \beta$ is negative which introduces $180^{\circ}$ phase change between $\left(\dot{m}^{\prime}, \dot{Q}_{r}^{\prime}\right)$. In both cases, the $180^{\circ}$ phase change for $\beta<0$ and $\alpha>0$ may trigger a thermoacoustic instability near the blow-out limit, as shown next.

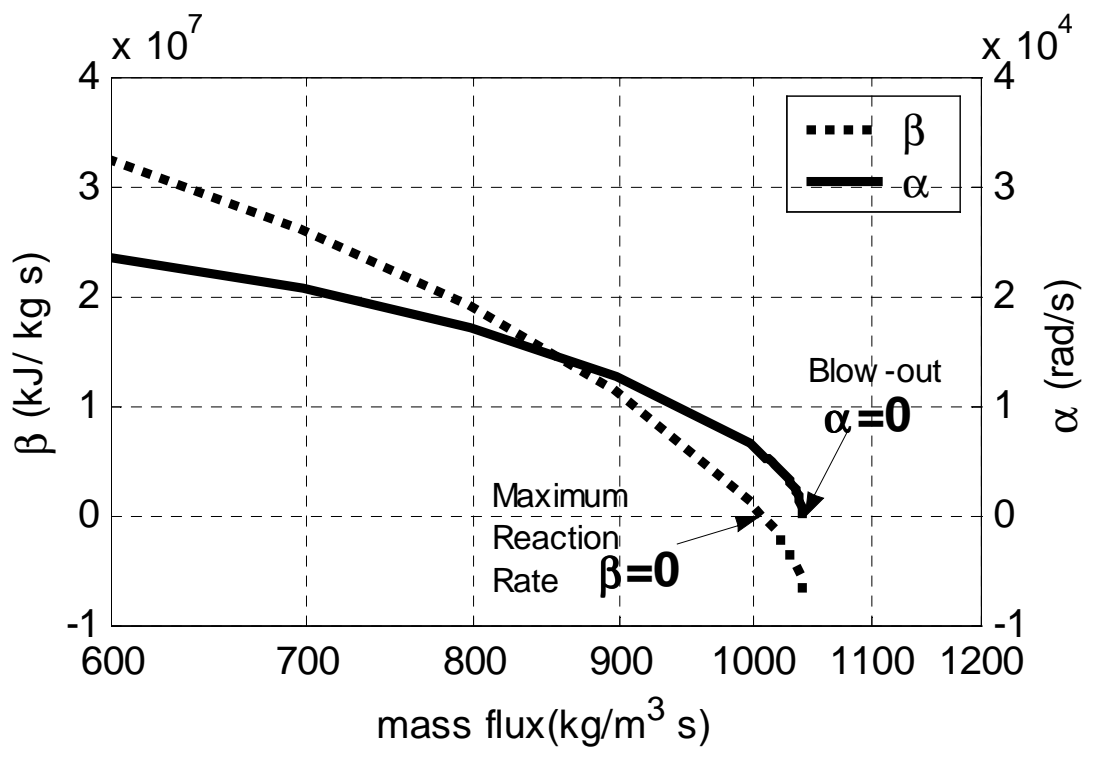

Figure 5 The dependence of the cut-off and static gain on the mass flow rate at $\phi=0.8$. 


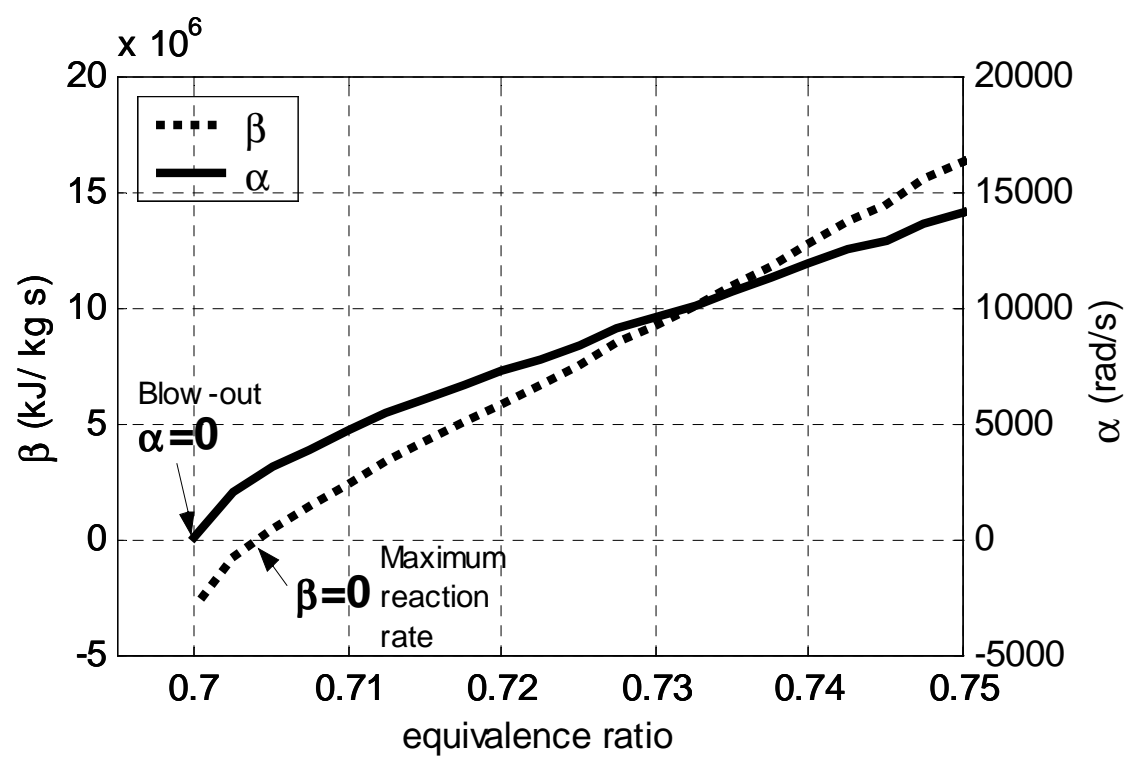

Figure 6 The dependence of the cut-off and static gain on the equivalence ratio at $\dot{\bar{m}} / V=530 \mathrm{~kg} / \mathrm{m}^{3}$.

The characteristics of the heat release model are exhibited in Figures 7-10 at a given acoustic frequency, e.g., $200 \mathrm{~Hz}$. The phase between $\dot{m}_{i}^{\prime}$ and $\dot{Q}_{r}^{\prime}$ changes from $0^{\circ}$ at low $\dot{\bar{m}}_{i}$ to small negative values as we approach $T^{* *}$ (the point of the maximum heat release) as shown in Figure 7. A $180^{\circ}$ increase in phase is experienced at $T^{* *}$. For $\dot{\bar{m}}_{i}$ corresponding to $T^{*}<T<T^{* *}$, the phase decreases to $90^{\circ}$. The sudden phase jump at the maximum heat release point corresponds to the sign change of $\beta$, while the continuous phase change is due to the decrease of $\alpha$. Figure 8 shows the dependence of the gain on $\dot{\bar{m}}$. Note the sharp increase for $T<T^{* *}$. 


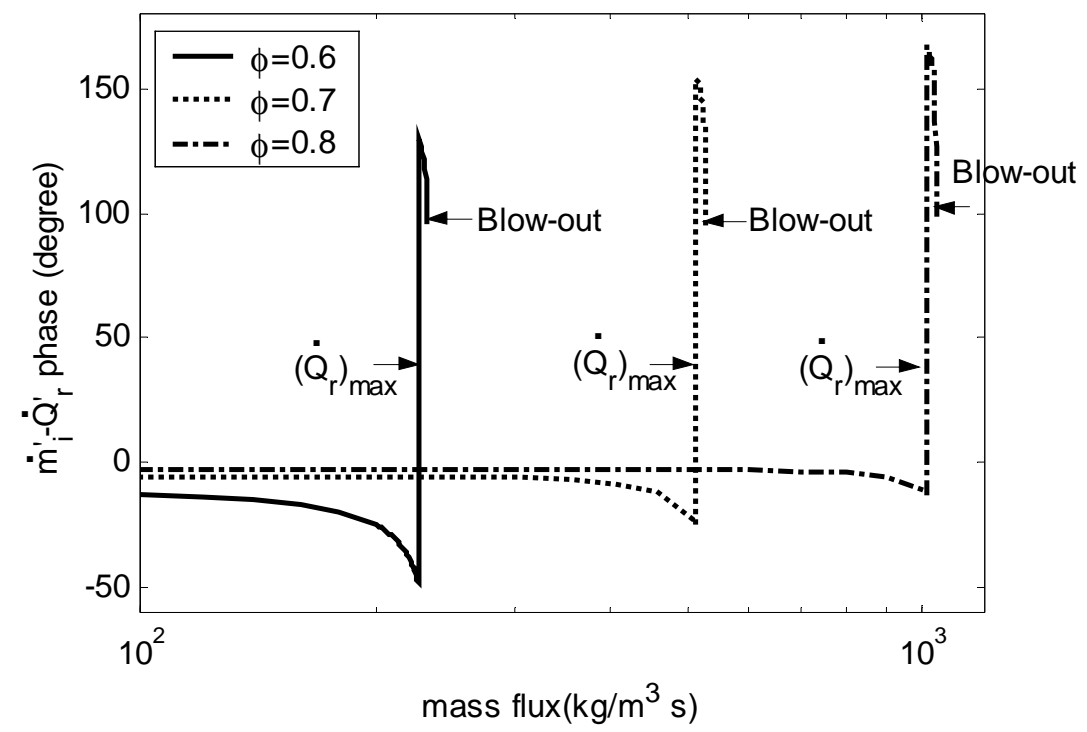

Figure 7 Dependence of the phase of the heat release model on the mass flow rate at different equivalence ratios

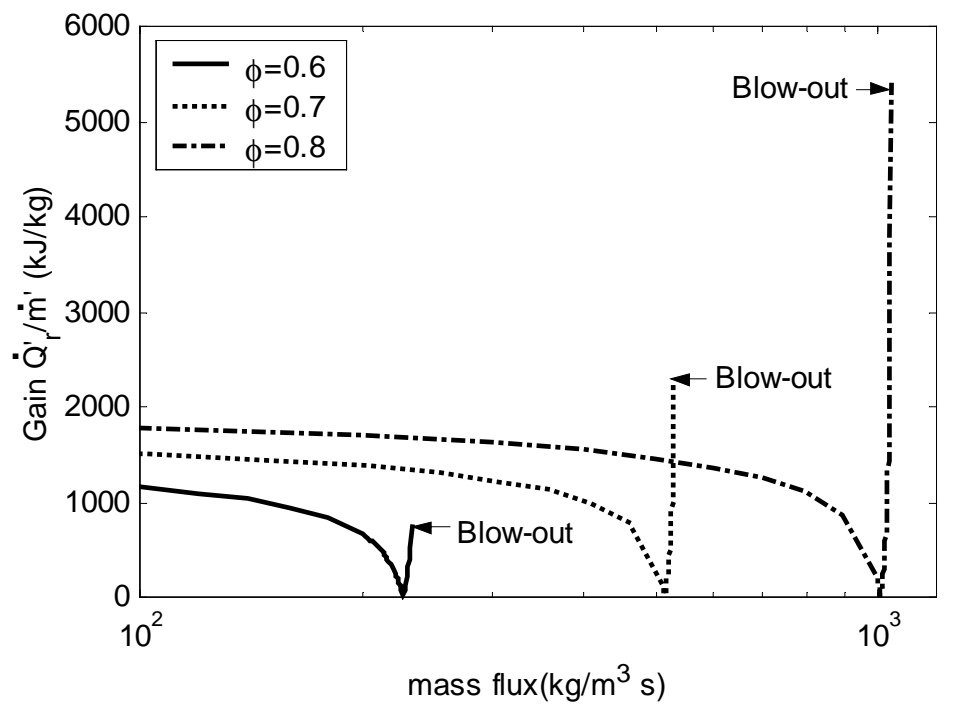

Figure 8 Dependence of the gain of the heat release model on the mass flow rate at different equivalence ratio 


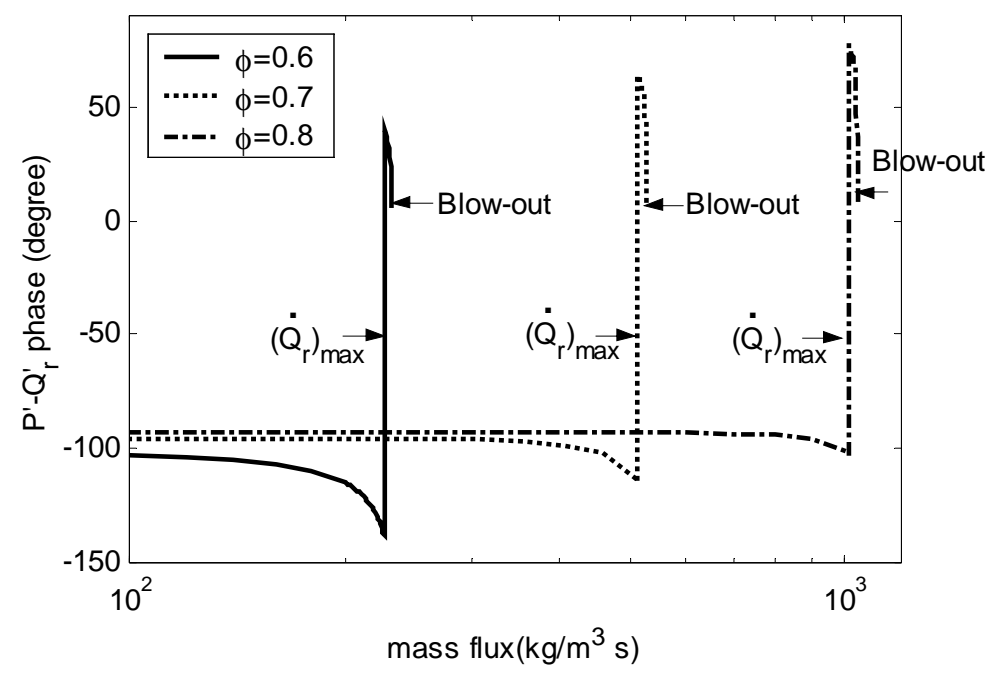

Figure 9 Dependence of the $p^{\prime}-\dot{Q}_{r}^{\prime}$ phase on the mass flow rate for a quarter-wave mode using the heat release model in Figure 7 and 8

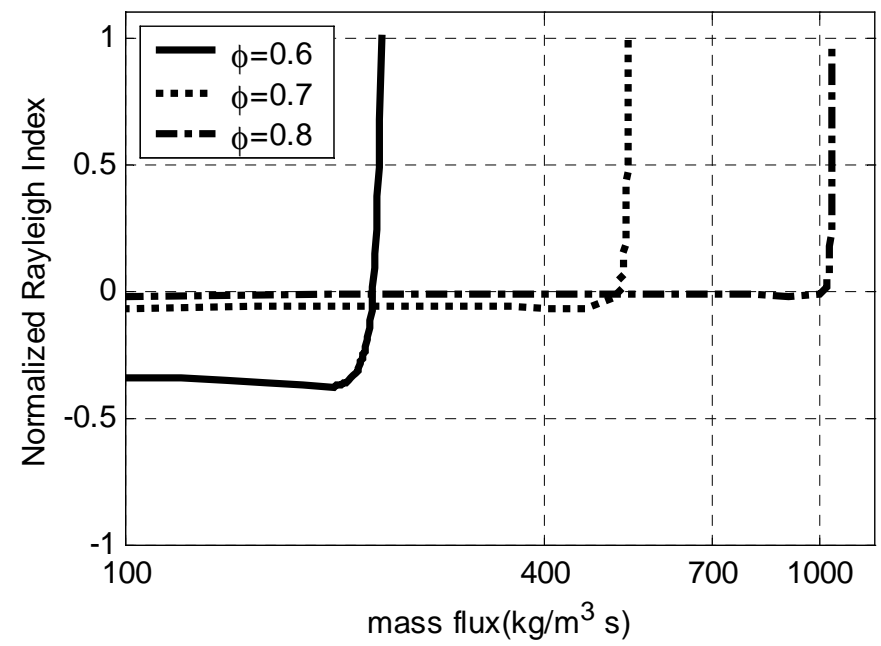

Figure 10 The Rayleigh Index for a quarter-wave mode using the heat release model in Figure 8 and 9. 


\section{Heat Release Dynamics-Acoustics Coupling}

The possibility that a phase change of $180^{\circ}$ at $T^{* *}$ may trigger a thermoacoustic instability is now demonstrated. To model the latter, we must determine the $p^{\prime}-v^{\prime}$ relationship. The momentum equation shows that the phase between $p^{\prime}$ and $v^{\prime}$ (inlet velocity perturbation) is $90^{\circ}$. In open-closed boundary conditions, the first two longitudinal acoustic modes correspond to a quarter-wave and a three-quarter-wave. For a quarter-wave mode, $p^{\prime}$ leads $v^{\prime}$ over the entire combustor, i.e. $\angle p^{\prime}-v^{\prime}=-90^{\circ}$. For a three-quarter-wave, $p^{\prime}$ leads $v^{\prime}$ on either sides of the left and right nodes, $\angle p^{\prime}-v^{\prime}=-90^{\circ}$, while $v^{\prime}$ leads $p^{\prime}$ between the two nodes, $\angle p^{\prime}-v^{\prime}=90^{\circ}$. Moreover, $\dot{m}^{\prime}$ can be represented as

$$
\dot{m}^{\prime}=\rho_{i} v^{\prime} A
$$

where $\rho_{i}$ is the density and $A$ is the cross sectional area of the combustor. Using the heat release model, the phase $\angle p^{\prime}-v^{\prime}$ and the relation in equation (22), the phase between $p^{\prime}$ and $\dot{Q}_{r}^{\prime}$ can be determined. Figure 9 shows $p^{\prime}-\dot{Q}_{r}^{\prime}$ phase as a function of $\dot{\bar{m}}$ for three different equivalence ratios, assuming a quarter-wave mode for the $p^{\prime}-v^{\prime}$ relation. It shows that $p^{\prime}$ and $\dot{Q}_{r}^{\prime}$ are in-phase between the point of maximum heat release rate and the blow-out limit. As discussed in Section I, thermoacoustic instabilities occur when $p^{\prime}$ and $\dot{Q}_{r}^{\prime}$ are in-phase. Moreover, as shown in Figure 8, as the mass flow rate increases, the gain decreases first reaching a minimum at $T^{* *}$, and then increases again. Both effects indicate that one should expect strong pressure oscillation near the blow-out limit. 
Given the magnitude and the phase relation as shown in Figure 8 and 9, it is possible to compute the Rayleigh Index, $I_{R}$, which is defined as

$$
I_{R}=\iint p^{\prime} q^{\prime} d t d V
$$

where $q^{\prime}=\dot{Q}_{r} / V$. Positive values of $I_{R}$ lead to strong pressure oscillation, whereas negative $I_{R}$ indicates a stable system. Figure 10 shows the Rayleigh Index normalized by its maximum value at the same conditions shown in Figure 8 and 9. The Rayleigh Index experiences a sharp increase between the point of maximum heat release and blow-out as the mass flow rate increases. The maximum Rayleigh Index is achieved at the blow-out point.

Figure 11 shows the impact of the equivalence ratio on $\left(p^{\prime}, \dot{Q}_{r}^{\prime}\right)$ gain and phase relations. Near blow-out, $p^{\prime}$ and $\dot{Q}_{r}^{\prime}$ become in-phase while their gain increases sharply. Note the narrow range of $\phi$ within which conditions support an instability.

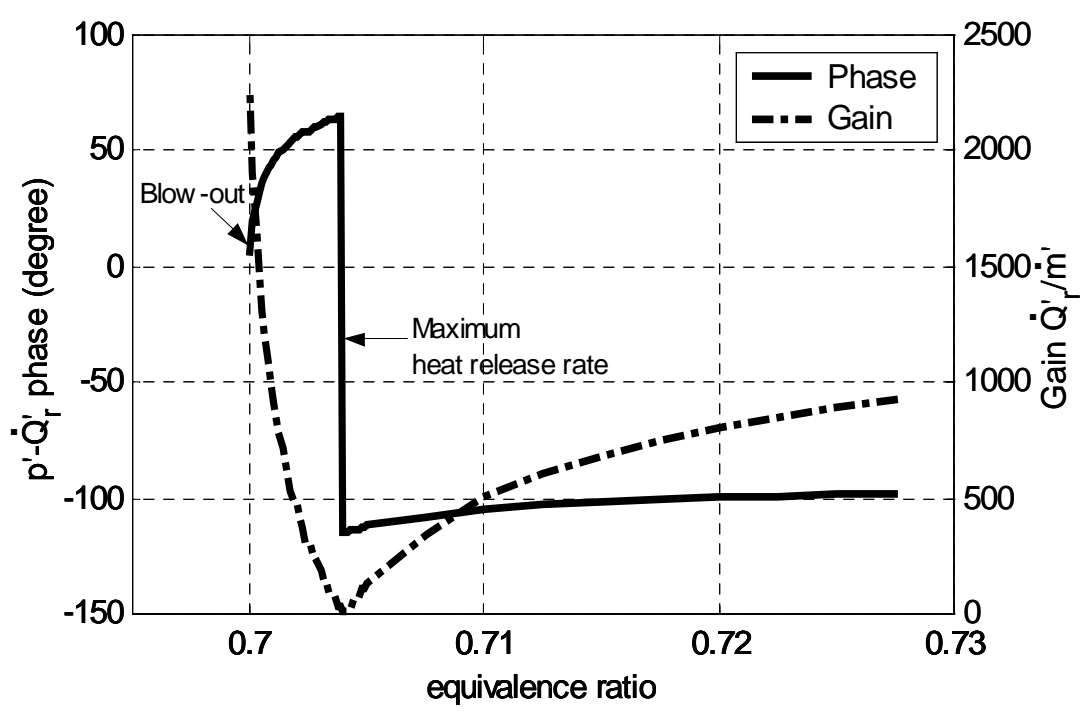

Figure 11 The $\left(p^{\prime}-\dot{Q}_{r}\right)$ phase and gain for a quarter-wave mode at a fixed mass flow rate $\left(530 \mathrm{~kg} / \mathrm{m}^{\wedge} 3 \mathrm{~s}\right)$, as a function of the equivalence ratio. 


\section{Experimental Evidence}

There exists ample experimental evidence that as the equivalence ratio is decreased at a fixed mass flow, or the mass flow rate is increased as a fixed equivalence ratio, the system develops self-sustained oscillations. Soon after these oscillations are observed, blow-out is often encountered. In this section, we review some of these results and use the theory developed in this paper to explain some of concomitant observations.

In an experiment conducted to examine the response of a lean premixed, swirl stabilized combustor [8], it was observed that the system remained stable until rather low values of $\phi$, where thermoacoustic instabilities seem to become strong. Soon after the onset of the instability, and within a small decrease in $\phi$, combustion blows out in a way that is qualitatively similar to the prediction in Figure 11.

Results of a lean premixed combustor in which a flame was stabilized behind a rearward-facing step [9] exhibited the dependence of the pressure amplitude on the equivalence ratio shown in Figure 12. As the equivalence ratio decreased, the amplitude of a $48 \mathrm{~Hz}$ mode increased, while that of a $124 \mathrm{~Hz}$ mode decreased within the same range. According to the system configuration in [9], the $48 \mathrm{~Hz}$ mode corresponded to a quarter-wave mode, while the $124 \mathrm{~Hz}$ mode corresponded to a three-quarter-wave mode. The theory presented in this paper predicts this mode selective behavior, as shown below. Since the flame was located in the middle of the combustor in [9], $p^{\prime}$ leads $v^{\prime}$ in the 
quarter-wave mode while $v^{\prime}$ leads $p^{\prime}$ in the three-quarter-wave mode, as mentioned above. This in turn implies that at a given $\phi$, if $p^{\prime}$ and $\dot{Q}_{r}^{\prime}$ are in-phase in one mode, they are out-of-phase in the other. At the same time, the results of the previous section show that, for a given mode, the $p^{\prime}-\dot{Q}_{r}^{\prime}$ phase goes through a sudden change of $180^{\circ}$ as $\phi$ changes. Together these two facts lead to the observation that if one mode, say the $96 \mathrm{~Hz}$ mode, is stable and another mode, say the $124 \mathrm{~Hz}$ mode, is unstable at a given $\phi$, the stability can switch between the two modes as $\phi$ is decreased. We should mention that this agreement is only qualitative since the heat release dynamics in the experiment may be governed by flame surface motion. However, since the chemical time scale governs the heat release rate near the lean blow-out limit, the combustion dynamics can be approximated by a well-stirred reactor in that region. Note that the pressure amplitudes increase sharply prior to blow-out, as captured by the WSR model.

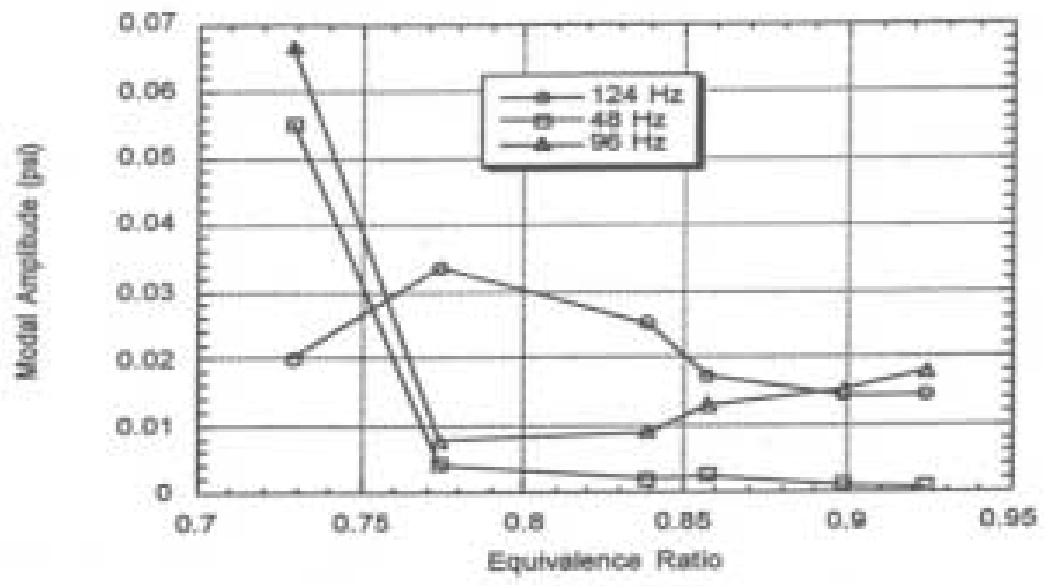

Figure 12 Pressure amplitudes in a lean premixed combustor near the blow-out conditions [9] 
The experimental results of Richards et al. [3] also agree with the prediction of the WSR model. In that study, the combustor used to investigate the effect of the heat loss, flow rate and friction was composed of a choked inlet, well-mixed combustion zone and a tail pipe. Because the inlet was choked, equivalence ratio fluctuations were absent. As shown in Figure 13, the pressure amplitude increased as the equivalence ratio was decreased at a fixed residence time $(39 \mathrm{~ms})$. Figure 14 shows the impact of the residence time at a fixed equivalence ratio. As the residence time was decreased (by increasing the mass flow rate), the pressure amplitude increased. The dependence of the stability of the system on the equivalence ratio and the residence time qualitatively match the predictions based on the WSR heat release dynamics model. Figure 13 and 14 also show that the mode changes to a lower frequency as the pressure amplitudes grow. This may be due to our prior observation that different phase relations for $\angle p^{\prime}-\dot{m}^{\prime}$ should be considered for different modes, and that the phase strongly depends on $\phi$ and $\dot{\bar{m}}$ through the model parameters $\alpha$ and $\beta$.

Another set of experimental result where a three-nozzle sector combustor was used with full-scale engine hardware [10] to examine the characteristics of an annular combustors showed sharp rise of pressure oscillation within the narrow range of equivalence ratios between 0.41 and 0.42 as shown in Figure 15. This is similar to the simulation result of the WSR model as shown in Figure 11.

In summary, these experimental studies support the following characteristics of the heat release dynamics model: 
1) As the equivalence ratio decreases or the mass flow rate increases, the system becomes unstable. The transition seems abrupt.

2) The instability is due to a sudden phase change near the lean blow-out limit. While the gain increases there as well, it cannot explain mode switching.

3) The combustion instability region is narrow ( $\Delta \phi \approx 0.1)$, and exists just before the lean blow-out.

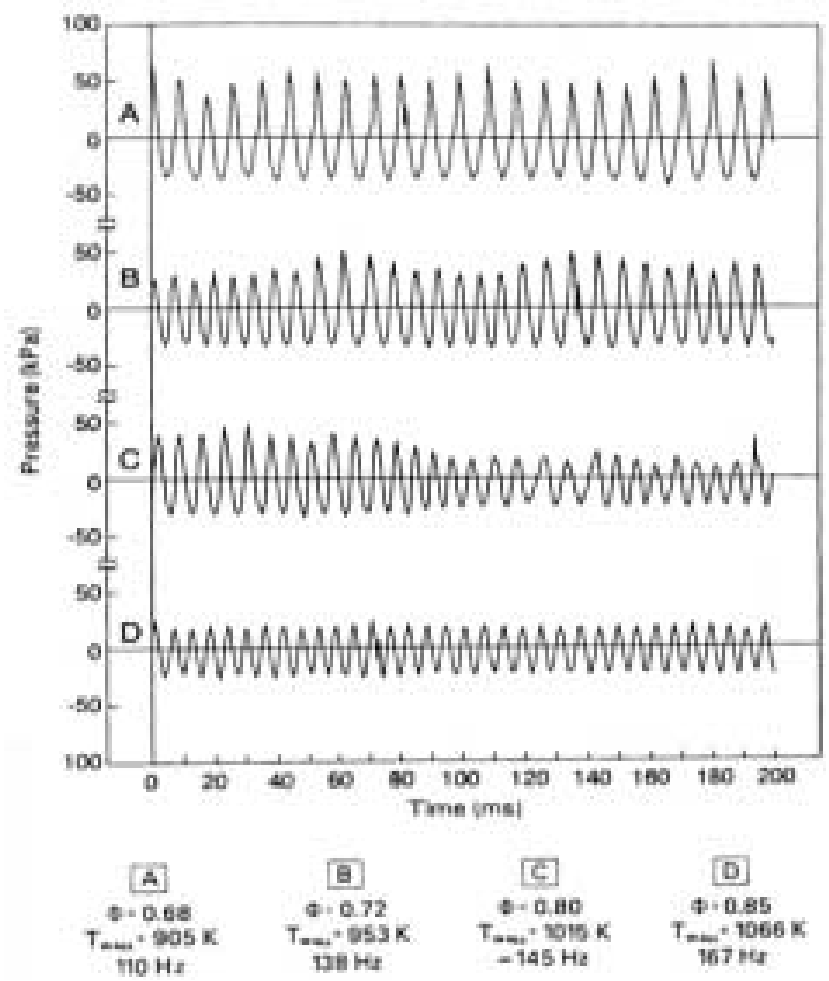

Figure 13 Pressure amplitudes in a combustor at various equivalence ratios [3] 


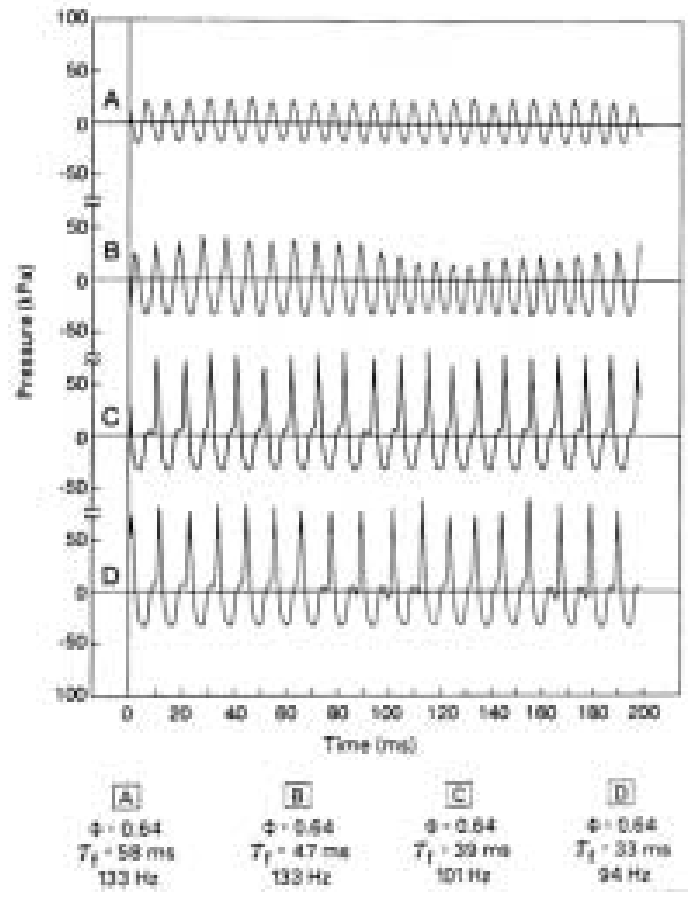

Figure 14 Pressure amplitudes in a combustor at various flow times [3]

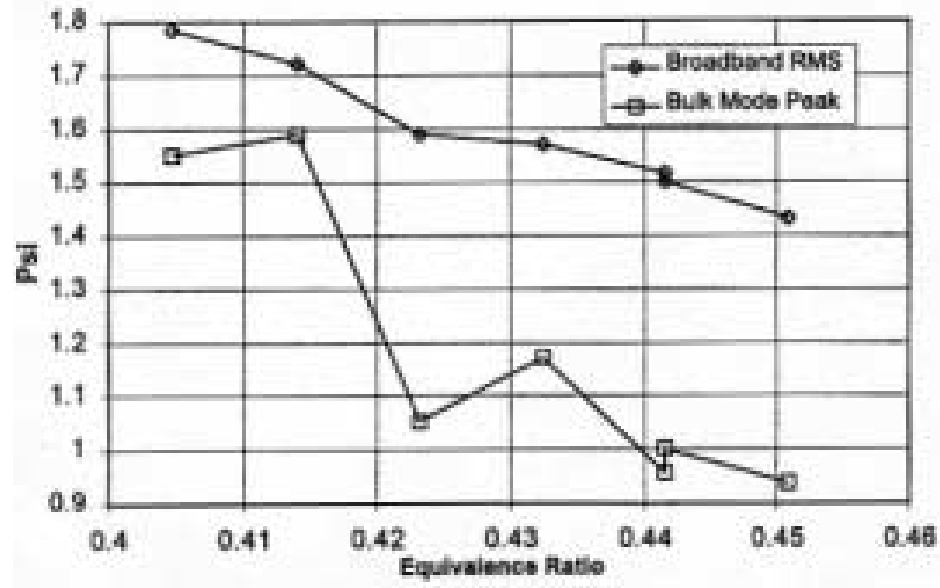

Figure 15 Change of pressure amplitudes near the lean blow-out limit [10] 
We observed that the system follows the characteristics of WSR model as shown in Figures 12-15. It is also necessary to examine whether the system can be represented by WSR model in those operating conditions to confirm the applicability of WSR model. One can determine the validity of the WSR model in a particular condition using two parameters in the phase plane, e.g., Damkohler number, $D_{a}$, and normalized turbulence intensity, $u^{\prime} / S_{u}$, where $u^{\prime}$ is the magnitude of the turbulence fluctuations in velocity $[11,12] . D_{a}$ is given by

$$
D_{a}=\frac{\tau_{t}}{\tau_{r}}=\frac{L / u_{t}}{\rho / \omega}
$$

where $\tau_{t}$ is the time scale of turbulence, $\tau_{r}$ is the time scale of combustion, $L$ is the characteristic length of a combustor, $u_{t}$ is turbulence velocity and $\omega$ is mass rate of formation. To justify using the WSR model in a particular operating condition, the following conditions should be satisfied:

$$
O\left(D_{a}\right)<1 \text { and } O\left(u^{\prime} / S_{u}\right)>1
$$

One can estimate $u^{\prime}$ from $p^{\prime}$ using the following equation:

$$
u^{\prime} \approx p^{\prime} /(\rho \cdot c)
$$

which is derived from the conservation equations. $p^{\prime}$ was 0.4 Psi in Ref [9] at $\phi=0.73$. Assuming $T=1700 \mathrm{~K}$, we get $u^{\prime} \approx 15.7 \mathrm{~m} / \mathrm{sec}$. Considering that $O\left(S_{u}\right) \approx 1 \mathrm{~m} / \mathrm{s}$, we get $u^{\prime} / S_{u}$ to be order of 10. For $\tau_{t}$, the length of the step height $(L=0.025 m)$ is used and $u_{t} \approx u^{\prime}$ is assumed. It gives $\tau_{t}=1.6 m \mathrm{sec}$. To calculate $D_{a}$, it is necessary to determine $\tau_{r}$ which strongly depends on temperature and mass ratio of species. Due to insufficient information, it is difficult to calculate exact $\tau_{r}$. Instead, one can approximately estimate 
the chemical reaction time using experimental data of hydro-carbon fuel. Figure 16 shows the chemical reaction time as a function of the equivalence ratio. It is shown that below $\phi<0.8$, the chemical reaction time decreases drastically. Near the blow out limit, $\tau_{r}$ becomes larger than $1.6 \mathrm{msec}$, which gives $D_{a}<1$. However, we observe that the chemical reaction time is much smaller than the turbulence time scale $\left(O\left(D_{a}\right) \approx 0.1\right)$ at stoichiometry. Therefore, it suggests that the WSR mode is applicable near the blow-out limit where the chemical time scale increases drastically, but it is not applicable at stoichiometry. It agrees with the results in section II.2 that the dynamics of the chemical reaction is negligible at stoichiometry where the time constant, $\alpha$, of the WSR model is much smaller than other time scales. For ref [3] and [10], as shown in Figures 13 to 15, the turbulence intensity is much larger than Ref [9] (One can expect it from the magnitude of the pressure oscillations) and the equivalence ratio is lower. Therefore, it is also reasonable to assume that those systems as WSR near the blow out limit.

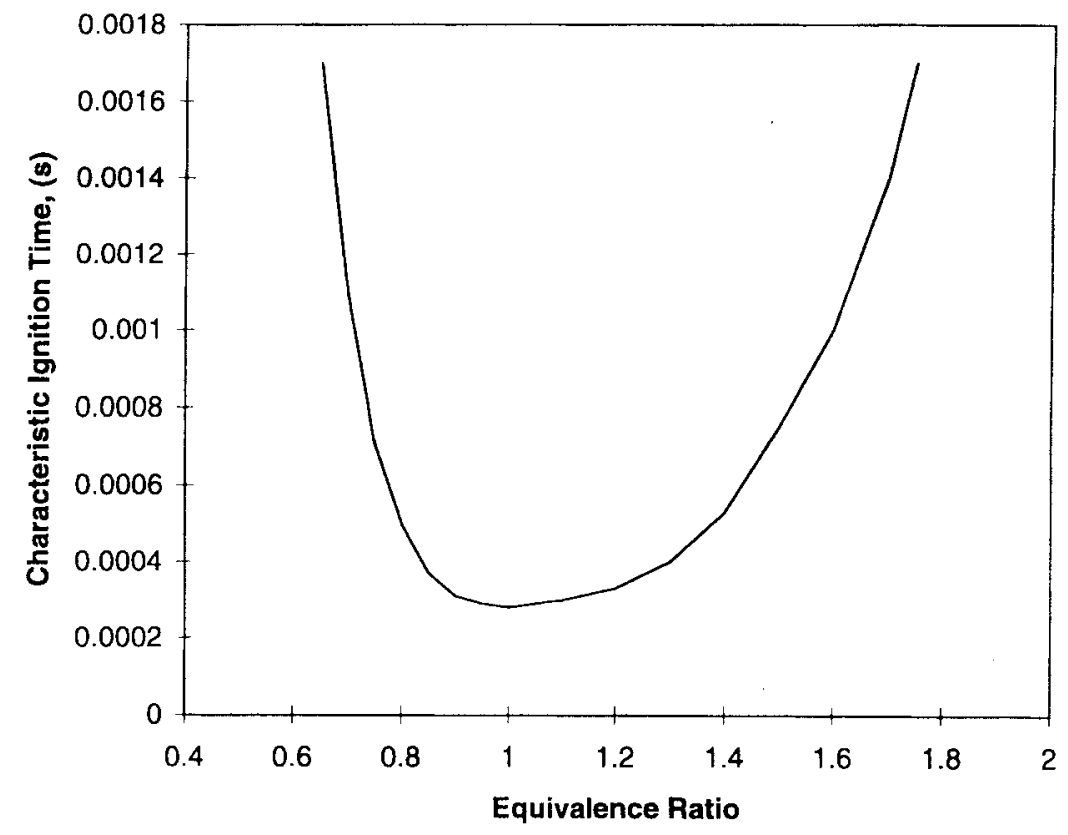

Figure 16 Characteristic chemical time for a hydrocarbon fuel [13] 


\section{Thermoacoustic Instability Simulations}

The model presented in Section II can be used to predict combustion instability once an acoustic model is derived. Using a Galerkin approximation [14-15], we express the unsteady pressure $p^{\prime}$ as:

$$
p^{\prime}(x, t)=\bar{p} \sum_{i=1}^{n} \psi_{i}(x) \eta_{i}(t)
$$

where $\psi_{i}(x)$ and $\eta_{i}(t)$ are modal shape and amplitude. Assuming that one acoustic mode is dominant, and that the heat release is localized at $x=x_{f}$, the amplitude this mode can be shown to be governed by (see Ref. [16]):

$$
\frac{d^{2} \eta}{d t^{2}}+\omega^{2} \eta=\gamma a_{o} E^{-1} \psi\left(x_{f}\right) \frac{d \dot{Q}_{r}^{\prime}}{d t}
$$

where $\omega$ is the acoustic frequency, $a_{o}=\frac{\gamma-1}{\bar{p}}$ and $E=\int_{0}^{L} \psi(x)^{2} d x$. Using the configuration of the LSU-swirl stabilized combustor [17], in which $\omega=1257 \mathrm{rad} / \mathrm{s}$ for a quarter mode, $L=0.6 m, \quad x_{f}=0.03 m, A=0.0196 \mathrm{~m}^{2}, \gamma=1.4, \quad \rho_{i}=0.6 \mathrm{~kg} / \mathrm{m}^{3}, \quad k=2.618$ and $\bar{p}=1 \mathrm{~atm}$, the following acoustic model is obtained

$$
\eta=F(s) \dot{Q}_{r}^{\prime}=\frac{0.0133 s}{s^{2}+1.579 \times 10^{6}} \dot{Q}_{r}^{\prime}
$$

The feedback relationship between $\dot{Q}_{r}^{\prime}$ and $p^{\prime}$ can be obtained as follows: The dependence of $\dot{Q}_{r}^{\prime}$ on $\dot{m}^{\prime}$ can be expressed using equation (15). Moreover the 
relationship between $\dot{m}^{\prime}$ and $p^{\prime}$ can be expressed using the momentum equation and equation (22) (see Ref. [16])

$$
\dot{m}^{\prime}=\rho_{i} A v^{\prime}=\left.\rho_{i} A \frac{1}{\gamma} \frac{d \psi}{d x}\right|_{x_{f}} k^{-2} \frac{d \eta}{d t}
$$

where $\rho_{i}$ is the density, $A$ is the cross sectional area of the combustor, and $k$ is the wave number. Using the data of the LSU combustor, we get

$$
\dot{m}^{\prime}=-2.512 \times 10^{-4} \frac{d \eta}{d t} .
$$

The parameters $\alpha$ and $\beta$ in the heat release model are evaluated for two different operating conditions. In both cases, $T_{i}=600 \mathrm{~K}$ and $\phi=0.6$, while for

Case I. $\dot{\bar{m}}_{i} / V=100 \mathrm{~kg} / \mathrm{m}^{3} \mathrm{~s}$,

$$
J(s)=\frac{6.6 \times 10^{6}}{s+5594}
$$

and to

$$
\begin{aligned}
& \text { Case II. } \dot{\bar{m}}_{i} / V=230 \mathrm{~kg} / \mathrm{m}^{3} \mathrm{~s}, \\
& J(s)=\frac{-3.475 \times 10^{5}}{s+746.5} .
\end{aligned}
$$

Using equations (24), (26), and heat release models in (27) and (28), we develop the combustion feedback system shown in Figure 17. For the given data, the maximum reaction is at $T^{* *}=1605 \mathrm{~K}$, while the blow-out is at $T^{*}=1555 \mathrm{~K}$. The equilibrium temperature is $1815 \mathrm{~K}$ in Case I and $1588 \mathrm{~K}$ in Case II. Note that the equilibrium temperature is $T^{* *}<T$ in Case I, while $T^{*}<T<T^{* *}$ in Case II. Figure 9 shows that in Case I $\angle p^{\prime}-\dot{Q}_{r}^{\prime}=-100^{\circ}$, and in Case II $\angle p^{\prime}-\dot{Q}_{r}^{\prime}=0^{\circ}$. Therefore, one can expect stable operation in Case I and pressure oscillation in Case II based on the Rayleigh Criterion. This is supported by the simulation results shown in Figure 18 and 19. 


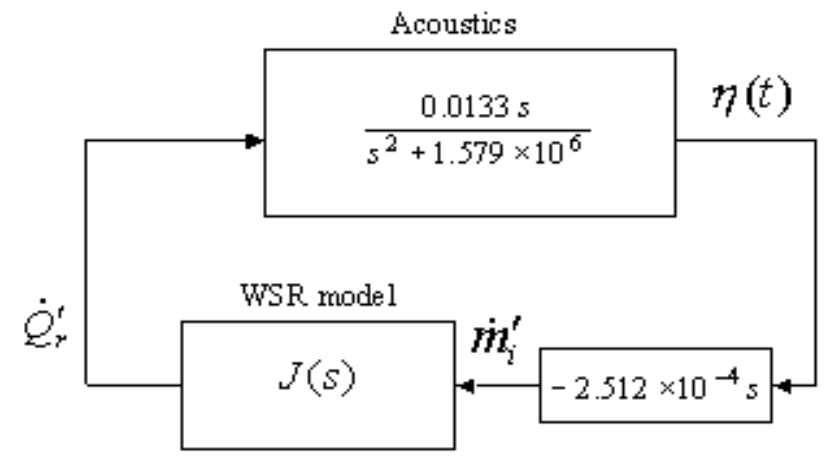

Figure 17 The combustion feedback system with the WSR model

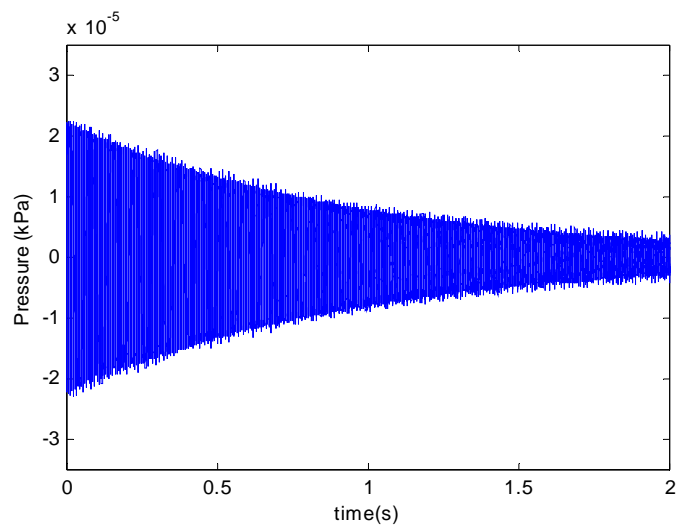

Figure 18 Simulation of pressure oscillation in Case I

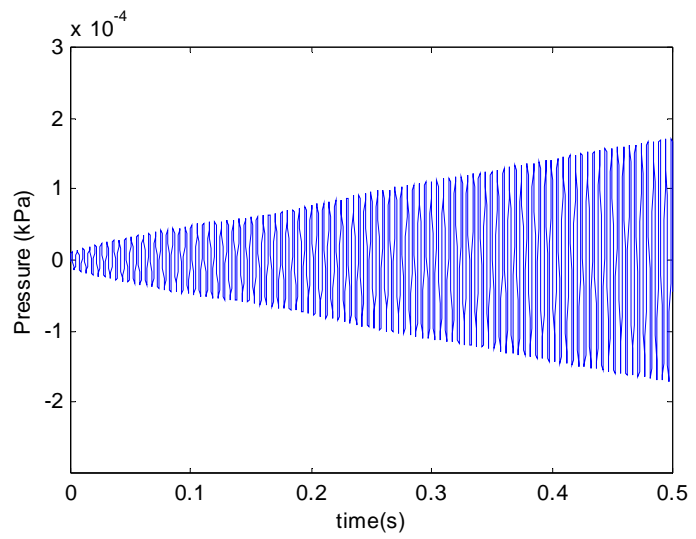

Figure 19 Simulation of pressure oscillation in Case II 
As shown in Figure 20, the same trend is observed in LSU experiment. As $\dot{\bar{m}}_{i}$ increases or $\phi$ decreases, the magnitudes of the pressure oscillations increase.

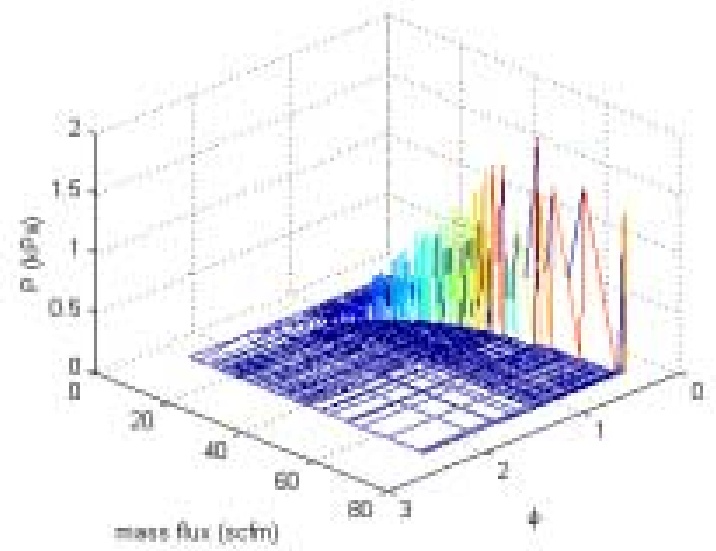

Figure 20 Pressure oscillation map in LSU swirl stabilized rig [17]

\section{Summary}

In this paper, we obtain a linearized heat release dynamics model based on the assumptions used in a well-stirred reactor, and express the heat release oscillation as a function of the mass flow rate. We limit the analysis to the case of single-step kinetics. The heat release dynamics model has the form of a first order filter, having a pole and a static gain. The model captures static blow-out as the pole becomes unstable, and shows that the phase between mass flow rate and the heat release oscillations changes by $180^{\circ}$ at the point of the maximum hear release, corresponding to the change of the sign of the gain. 
The phase and gain between mass flow oscillation and heat release perturbation depend on the mean residence time and equivalence ratio. Phase change occurs soon before blow-out. For certain cases, while it depends on the nature of the acoustic mode and the location of the heat release zone, the phase between $\left(p^{\prime}, \dot{Q}_{r}^{\prime}\right)$ changes from about $-90^{\circ}$ before the maximum reaction point to about $+90^{\circ}$, following a transition across this point, to around $0^{\circ}$ at blow-out as the residence time or the equivalence ratio is decreased. Based on the Rayleigh Criterion, the combustor may become unstable due to the positive coupling between the heat release dynamics and acoustics at the maximum power, or at lean burn condition close to lean blow-out. Experimental studies $[3,9,10$ 17] show similar characteristics.

We are currently extending the formulation of the heat release model to the case of a multi-step kinetics mechanism, which can yield more accurate model over a wide-range of operating condition.

\section{Acknowledgments}

This work is sponsored by the National Science Foundation, grant no. ECS 9713415, and the Office of Naval Research, grant no. N00014-99-1-0448. 


\section{$\underline{\text { References }}$}

1. Y.B Zeldovich, G. I. Barenblatt, V. B. Librovich and G. M. Makhviladze, "The mathematical theory of combustion and explosions," Consultant Bureaum, NY, 1985.

2. A. A. Putnam, "Combustion driven oscillations in industry," American Elsevier Publishing Company, NY, 1971.

3. G.A. Richards, G. J. Morris, D. W. Shaw, S. A. Keeley and M. J. Welter, "Thermal pulse combustion,” Combust. Sci. and Tech., Vol. 94, pp.57-85, 1993.

4. M. C. Janus and G. A. Richards, "Results of a model for premixed combustion oscillations," in the Proceedings of the 1996 AFRC International Symposium, Baltimore, MD, 1996.

5. T. Lieuwen, Y. Neumeier and B. T. Zinn., "The Role of unmixedness and chemical kinetics in driving combustion instabilities in lean premixed combustors," Combust. Sci. and Tech., Vol. 135, pp.193-211, 1998.

6. T. Lieuwen and B. T. Zinn, "Theoretical investigation of combustion instability mechanisms in lean premixed gas turbines," $36^{\text {th }}$ Aerospace Sciences meeting \& exhibit, Reno, NV, Jan 12-15, 1998. 
7. C. K. Westbrook and F. L. Dryer, “ Chemical kinetic modeling of hydrocarbon combustion,” Prog. Energy Combust. Sci., Vol.10, pp 1-57, 1984.

8. W. M. Proscia, Private Communication.

9. J. M. Cohen and T. J. Anderson, "Experimental investigation of near-blowout instabilities in a lean, premixed step combustor," $34^{\text {th }}$ Aerospace Sciences meeting \& exhibit, Reno, NV, Jan 15-18, 1996.

10. J. R. Hibshman, J. M. Cohen, A. Banaszuk, T. J. Anderson and H. A. Alholm, “ Active control of combustion instability in a liquid-fueled sector combustor," $44^{\text {th }}$ ASME Gas Turbine and Aeroengine Technical Congress, Indianapolis, IN , June 7-10, 1999.

11. R. Borghi, On the structure and morphology of turbulent premixed flames, in C. Casci, editor, Recent Advances in the Aerospace Science, pp.117-138, Plenum, N.Y.,1985.

12. T. J. Poinsot, D. Veynante and S. Candel, "Diagram of premixed turbulent combustion based on direct simulation," Twenty-third symposium (International) on combustion, pp.613-619, 1990.

13. Zukoski, E. Afterburners, In:Ogates G. (Ed.) The aerodynamics of aircraft gas turbine Engines, Air Force Propulsion Lab, Chapter 21, 1978. 
14. B. T. Zinn, and M. Lores, "Application of the galerkin method in the solution of nonlinear axial combustion instability problems in liquid rockets," Combust. Sci. and Tech., 4, pp. 269-278, 1972.

15. F. Culick., "Nonlinear behavior of acoustic waves in combustion chambers", Acta Astronautica, Vol.3, pp.715-756, 1976.

16. A. M. Annaswamy, M. Fleifil, J. P. Hathout, and A. F. Ghoniem, “ Impact of linear coupling on the design of active controllers for the thermoacoustic instability", Combust. Sci. and Tech., Vol. 128, pp.131-180, 1997.

17. S. Murugappan, S. Acharya, E. J. Gutmark and T. Messina, "Characteristics and control of combustion instabilities in a swirl-stabilized spray combustor", $35^{\text {th }}$ AIAA/ASME/SAE/ASEE Joint Propulsion Conference and Exhibit, L. A., CA, Jun 2024, 1999. 